\title{
Sentidos en disputa sobre la violencia hacia las mujeres en las políticas públicas. El caso de la normativa de la provincia de Córdoba, Argentina
}

Senses in dispute on violence against women in public policy. The case of the law of the province of Cordoba, Argentina

\author{
Maite Rodigou', Carlos Javier López² \\ Pamela Ceccoli2, Ivana Puche ${ }^{2}$, Valeria Aimar ${ }^{2}$
}

\begin{abstract}
Resumen
La violencia de género como problema social se ha instalado hace escasamente tres décadas en Argentina, a partir de los debates impulsados por el activismo feminista y los movimientos de mujeres en el reconocimiento de sus derechos. Desde estas acciones y demandas, se generan normas, legislaciones, instituciones y recursos desde el Estado para atender esta problemática. Interesa en este artículo, producto parcial de una investigación cualitativa en curso, analizar los sentidos en disputa sobre la violencia hacia las mujeres que se presentaron entre el movimiento de mujeres y el Estado, respecto de la normativa que diera sustento a la política pública de la provincia de Córdoba, Argentina, en el período 2004-2008. La consideración y alcance de la violencia hacia las mujeres y el tipo de políticas previstas evidencian la tensión entre una concepción asistencialista, victimista y terapéutica, frente a una posición sustentada en el respeto y la garantía de los derechos humanos y de justicia.
\end{abstract}

Palabras clave: violencia hacia las mujeres - disputa de sentidos - políticas públicas - movimiento de mujeres - Estado.

\begin{abstract}
Gender violence as a social problem was established three decades ago in Argentina, from the debates driven by feminist activists and women's movements towards the recognition of their rights. These actions and claims generated rules, laws, institutions and resources from the State to address this issue. In this article, partial product of a qualitative research in progress, we aim at analyzing the senses in dispute about violence against women that appeared between women movements and the State, regarding rules that supported public policies in the province of Cordoba, Argentina, in 2004-2008. Consideration and reach of violence against women and the type of policies brought to light the tension between a victimized, therapeutical and welfared conception, compared to a position based on respect and warranty for human rights and justice.
\end{abstract}

Key words: violence against women - senses dispute - public policy- women's movement - State.

Licenciada en Psicología, Facultad de Filosofía y Humanidades. Universidad Nacional de Córdoba. Master en Estudios de las Mujeres, impartido por el Centre d'Investigació Histórica de la Dona, Universitat de Barcelona, España.

Profesores e investigadores, Universidad Nacional de Córdoba, Argentina. 


\section{INTRODUCCIÓN}

Los movimientos feministas y de mujeres vienen señalando y nominando la violencia por razones de género como una de las violaciones a los derechos humanos, en los últimos treinta años, buscando desnaturalizar los actos violentos que se originan, y denunciándolos. Se han ubicado así como interlocutores frente a la sociedad, y fundamentalmente frente al Estado y a las entidades supranacionales como las Naciones Unidas o la Organización de Estados Americanos, exigiendo leyes, normativas, estructuras y acciones de atención y prevención. Fueron estos movimientos los que han procurado instalar en la agenda pública la violencia hacia las mujeres como un problema de derechos humanos y de ciudadanía y, por tanto, como un asunto de justicia.

A partir de estas acciones y demandas, los Estados de la región latinoamericana -y Argentina no ha constituido excepción- han construido muy recientemente la violencia de género como "problema social" (Bosch y Ferrer, 2000; Marugán Pintos y Vega Solís, 2001 y 2002), creando normas, legislaciones, instituciones y recursos para atender la violencia hacia las mujeres, predominantemente. Dichas normativas y recursos, en general, se pueden analizar en tanto resultado y producto de las discusiones entre Estado y movimientos de mujeres y feministas, sin restar importancia al resto de actores sociales organizados.

Marugán Pintos y Vega Solís (2002), en el contexto español, señalan que en la conceptualización y tratamiento del problema de la violencia hacia las mujeres se pueden identificar cuatro momentos generados en esta interacción entre Estado y movimientos feministas: una primera etapa en donde la violencia no es nombrada como tal, una segunda etapa en la que es concebida en términos de derechos y libertades a partir de las acciones feministas, una tercera etapa en términos de delito y una cuarta en la que es considerada un problema de gestión. Para nuestra investigación, abordaremos esta identificación del tratamiento de la violencia hacia las mujeres en su aporte conceptual, y no en su desarrollo histórico-cronológico. Interesa desarrollar en nuestro análisis las tensiones que se establecen entre la consideración de la violencia como un problema de derechos y libertades y, por otra parte, como un problema de gestión y administración.

Ahora bien, las normas y políticas públicas creadas no siempre responden a las expectativas que presentan los movimientos de mujeres y feministas respecto de ellas. Distintos análisis sobre las políticas públicas que reconocen y buscan garantizar los derechos de las mujeres, señalan importantes limitaciones de las mismas. Entre estas limitaciones, y como rasgos comunes que poseen dichas políticas, Silvia Chejter (2009) va a plantear la fragilidad, discontinuidad, subalternidad, falta de integralidad, y presupuesto insuficiente. Además de estas características generales, las políticas públicas específicas que abordan la violencia hacia las mujeres presentan una concepción asistencialista, victimista y delegativa de la problemática y, en general, están centradas en la violencia intrafamiliar (Rainero, Rodigou, Pérez, 2005), desconociendo la magnitud del problema y el entramado social, cultural y político que lo sostiene y reproduce. 
Es así que las políticas desarrolladas, muchas veces, desvirtúan el sentido de las demandas históricas del feminismo en la consideración de la violencia hacia las mujeres como un problema de poder. Nancy Fraser (1991) ya señaló este último fenómeno, cuando analiza el momento en que los gobiernos locales de Estados Unidos se hicieron cargo del financiamiento y la administración de los albergues para mujeres maltratadas que habían iniciado hace tiempo grupos feministas, donde las mujeres pasaron de ser sujetos potentes -y feministas potenciales- a ser víctimas pasivas a las que había que ayudar. El problema se individualizó y psiquiatrizó, y el discurso se despolitizó, comenzando a utilizarse un lenguaje académico "neutral" para referir y explicar la violencia hacia las mujeres.

En nuestro continente latinoamericano también se ha discutido ampliamente -más en el terreno del activismo que en el académico- sobre la apropiación del discurso feminista por parte del Estado y la pérdida de su carácter subversivo (Sagot, 2008). La crítica feminista, por tanto, debe ir más allá de los reclamos por recursos insuficientes o de los déficits de algunos servicios, poniendo el foco de atención en los fundamentos de la política analizada y los resultados de las interacciones del movimiento con el Estado.

Al mismo tiempo, los movimientos feministas y de mujeres se encuentran con una paradoja en su accionar político, ya que si bien el Estado es su interlocutor principal para el efectivo reconocimiento de sus derechos, es el mismo Estado el que viola cotidianamente estos derechos, por acción u omisión, por lo que deben ejercer continuamente una función de monitoreo, vigilancia y denuncia ${ }^{3}$. Ante contextos estatales adversos a los derechos de las mujeres, las acciones de los movimientos feministas han tenido un carácter de denuncia radical, mientras que en contextos más favorables -cuando existe la llamada "voluntad política" de los gobiernos y/o funcionarios de turno- han adoptado formas críticas-propositivas, realizando recomendaciones que posibilitaron modificaciones en las políticas públicas implementadas. Es necesario recalcar que, a pesar de los riesgos existentes en la institucionalización de las propuestas feministas, las organizaciones de mujeres siguen trabajando en la construcción de políticas públicas respecto de la violencia de género, ya que se constituyen en recursos que posibilitan nuevas opciones de vida y derechos a las mujeres que viven violencia, como dice Sagot (2008).

Este artículo es producto de la investigación "Espacios y prácticas de organizaciones feministas y movimiento de mujeres. Sentidos en pugna sobre la violencia de género", desarrollada desde la Universidad Nacional de Córdoba, donde uno de los objetivos que nos planteamos fue reconstruir los sentidos sobre la violencia de género que sostiene el Estado provincial en sus normativas, discursos y mecanismos institucionales, a fin de establecer puntos de contacto y de conflicto con los planteamientos del activismo

Es frecuente que, a la hora de investigar y denunciar la violencia estatal, los análisis se vuelquen a la violencia que ejercen los agentes del Estado en las distintas áreas contra las mujeres, ya sea como violencia activa o por omisión, obviando la propia conformación del Estado con relación al sistema de géneros imperante, y la operatoria previa y simultánea a la vez del contrato sexual en todo contrato social, que señaló Pateman en su obra ya clásica "El contrato Sexual". 
feminista. El análisis gira en torno a la formulación de la política pública referida a esa problemática entre los años 2004 y 2008 en la provincia de Córdoba, Argentina.

Para ello, abordamos la documentación referida al anteproyecto de ley sobre violencia hacia las mujeres elaborado por una articulación de mujeres y organizaciones feministas y de mujeres de Córdoba, los debates en la legislatura provincial -a partir de las versiones taquigráficas de la mismas-y las normativas sancionadas sobre violencia familiar: Ley Provincial No 9283, su Reglamentación y Decreto 1030/08².

\section{LA ACCIÓN FEMINISTA Y DE MUJERES POR UNA VIDA SIN VIOLENCIA: EL DEBATE CON EL ESTADO}

Interesa señalar que la Ley No 9283 -Ley de Violencia Familiar-, sancionada el 10 de marzo de 2006, fue el producto final de una serie de debates que se originó en la presentación ante la legislatura provincial el 25 de noviembre de 2004 del "Anteproyecto de ley para prevenir, sancionar y erradicar la violencia hacia las mujeres en el ámbito familiar y las relaciones interpersonales", elaborado por el Movimiento de Mujeres Córdoba $(\mathrm{MMC})^{5}$.

Este anteproyecto de ley fue una construcción colectiva de mujeres de la provincia de Córdoba de diversos sectores, condiciones sociales y económicas, posicionamientos políticos, edades, ocupaciones, orientaciones sexuales, ante la necesidad de contar con una ley integral que prevenga, sancione y erradique la violencia hacia las mujeres. Dicha necesidad se fundamenta en una serie de razones esgrimidas desde el MMC. Principalmente podemos mencionar:

- La evidencia, a partir de una lectura de la realidad local, de que la violencia contra las mujeres es ejercida de manera cotidiana en una sociedad patriarcal y machista, y que la misma se encuentra naturalizada y, por tanto, invisibilizada su presencia y sus efectos.

- La ausencia de una legislación provincial, que específica y prioritariamente aborde la violencia contra las mujeres, mediante acciones de prevención, sanción y erradicación de dicha violencia.

$4 \quad$ Las fuentes documentales utilizadas fueron: "Anteproyecto de ley para prevenir, sancionar y erradicar la violencia hacia las mujeres en el ámbito familiar y las relaciones interpersonales". Movimiento de Mujeres Córdoba, 25 de noviembre de 2004, Versiones taquigráficas de las Comisiones de Solidaridad, Legislación General, Función Pública, Reforma Administrativa y Descentralización (período 2004-2005), Versión taquigráfica Sesión Ordinaria de la Legislatura de la Provincia de Córdoba el $1^{\circ}$ de marzo de 2006, Ley Provincial № 9283. De Violencia Familiar. Publicada en Boletín Oficial el 13 de marzo de 2006, Reglamentación de la Ley № 9283. Publicada en Boletín Oficial el 8 de marzo de 2007, y Decreto 1030/08 Programa de Prevención y Erradicación de la Violencia Familiar en la Provincia. Publicado en Boletín Oficial el 6 de noviembre de 2008.

5 Es importante recordar que un anteproyecto de ley puede ser presentado a la legislatura por cualquier institución, organismo no gubernamental, o por cualquier ciudadano o ciudadana. Pero quien toma ese proyecto debe ser un/a legislador/a para su tratamiento parlamentario. 
- La existencia de tratados y convenciones internacionales que especifican los compromisos que está obligado a cumplir el Estado argentino en todas sus jurisdicciones. Particularmente se alude a la Convención sobre la Eliminación de todas las formas de Discriminación contra la Mujer (CEDAW) de 1979, ratificada por Argentina en 1985 por la Ley N²31796 y la Convención Interamericana para Prevenir, Sancionar y Erradicar la Violencia contra la Mujer, Belem do Pará, en los artículos 7 y 8 . Dicha convención fue adoptada en la Asamblea General de la OEA en 1994, y ratificada por Argentina en el año 1996 por la Ley N²4.632². En tanto Argentina es un país federal, las provincias cuentan con sus legislaciones específicas.

Es de destacar, que previo a la construcción del anteproyecto, en el año 2003, el MMC elabora y presenta a la legislatura una documentación que contenía antecedentes de normativas nacionales e internacionales donde se ratificaba la necesidad de elaborar una ley en el sentido que se viene planteando. En dicho momento, esta demanda no obtuvo respuesta por parte de legisladores y legisladoras provinciales.

Ante este hecho, y sumado a las razones previamente planteadas, es que el MMC cambia su estrategia y decide de manera consensuada elaborar un anteproyecto de ley, afirmando su presencia activa, como mujeres integrantes de un movimiento social, en la construcción de políticas públicas garantes de los derechos de las mujeres. Desde el movimiento se hace una inversión del juego de la política tradicional: las mujeres dejan de ubicarse como objeto de políticas públicas a ser sujetos protagonistas de las mismas.

Para ello, el MMC realizó una serie de consultas a otros grupos y organizaciones feministas pertenecientes a distintas provincias, así como el estudio de diversas leyes de violencia familiar existentes en el país, abocándose especialmente a la ley de la provincia de Santa Fe ${ }^{8}$, teniendo como marco a la Convención de Belém do Pará.

Simultáneamente se realizaron actos públicos en fechas claves del movimiento de mujeres, tendientes a sensibilizar y concientizar con relación a la temática.

El proceso de construcción del anteproyecto implicó un gran esfuerzo, una presencia y participación activa permanente de las activistas del MMC, y finalizó en una plenaria donde se consensuaron los artículos contenidos en el mismo. Ya con el anteproyecto construido se decide presentarlo en la legislatura provincial el 25 de noviembre de 2004, una fecha emblemática para los movimientos de mujeres y feministas de la

$\overline{6}$ En 1994 se le otorga jerarquía constitucional junto a los demás tratados de DDHH en el art. 75 inc. 22 de la Constitución Argentina.

En septiembre del año 2011 se confiere también jerarquía constitucional a la Convención Belem do Pará.

8 La Ley Provincial No 11.529 de la Provincia de Santa Fe, Argentina, "De protección contra la violencia familiar", de 1998, fue tomada como modelo por el MMC para muchas de las formulaciones del anteproyecto de ley, ya que se la consideró como una de la más avanzadas en el país en temática de violencia familiar. 
región latinoamericana. Así lo relataban las integrantes del MMC en una reunión de comisiones de legislatura9?:

"... Entonces nos juntamos con mujeres víctimas de violencia y en noviembre del año pasado (se refiere al año 2004), logramos tener nuestra primer plenaria con estas expertas de Santa Fe y consensuamos con el resto de las compañeras del Movimiento de Mujeres todos los artículos..."

(Valentina Machietto, MMC)

"...vinimos en una manifestación callejera y lo dejamos en la Legislatura para que quedara claro que era un problema de toda la ciudadanía y no de un partido político. Este es el modo como trabajamos..."

(Patricia Triguero, MMC)

"...Para el día 25 de noviembre, Día Internacional de la Erradicación de la Violencia hacia las Mujeres, donde recordamos el asesinato de las tres hermanas Mirabal por la dictadura de Trujillo, en República Dominicana, presentamos el anteproyecto de ley en esta Legislatura. Previo a ello convocamos a todos los legisladores y legisladoras de la ciudad de Córdoba para que estuvieran presentes cuando se presentara ese anteproyecto pero, lamentablemente, sólo dos legisladoras y dos asesoras concurrieron a recibir el proyecto, el que fue ingresado por la Comisión de Solidaridad..."

(Valentina Machietto, MMC)

Aun sabiendo que no había antecedentes legislativos en el país y los posibles obstáculos que esto pudiera ocasionar, el MMC decide presentar este Anteproyecto que implicaba una innovación sustancial respecto de las leyes hasta el momento sancionadas. Asimismo, de haberse aprobado el "Anteproyecto de ley para prevenir, sancionar y erradicar la violencia hacia las mujeres en el ámbito familiar y las relaciones interpersonales", se habría producido un hecho histórico en Argentina: una ley elaborada por un movimiento social de mujeres.

En este punto, cabe aclarar que el anteproyecto elaborado por el MMC es presentado en la legislatura en el mes de febrero del 2005 por tres diputadas de la Unión Cívica Radical, como un anteproyecto de autoría del MMC, sin realizarle modificación alguna, y llamativamente, un mes después, en marzo del mismo año, una legisladora por el partido gobernante (Unión por Córdoba) dio ingreso a otro proyecto de ley pero relativo a violencia familiar. Ambos proyectos pasaron para su tratamiento a la Comisión de Solidaridad; sin embargo, solamente se trató el segundo proyecto (Ezpeleta, 2007).

En el tratamiento parlamentario del proyecto de ley de "violencia familiar", las presidencias de las Comisiones de Solidaridad (Comisión madre en el tratamiento de este proyecto), y de la Comisión de Legislación General propusieron al resto de los miembros

9 Las comisiones refieren a la forma organizativa que adopta la legislatura para el tratamiento del tema. Las expresiones de las integrantes del MMC han sido recuperadas de la versión taquigráfica de la reunión conjunta de las Comisiones de Solidaridad y Legislación General, Función Pública, Reforma Administrativa y Descentralización, Córdoba, 16 de agosto de 2005. Legislatura de la Provincia de Córdoba. 
legisladores de estas comisiones una metodología de trabajo que fue aceptada por unanimidad. La propuesta consistió en invitar a todas las partes que tenían interés en manifestar una opinión, o sus conocimientos profesionales, así como a todos los entes intermedios de esta sociedad que tenían alguna vinculación directa con este tema (asociaciones profesionales, organizaciones de la sociedad civil, etc.), para finalmente elaborar un despacho ${ }^{10}$.

Se realizan, así, al menos ocho reuniones de comisiones entre los años 2004, 2005 y $2006^{11}$, de las cuales las integrantes del movimiento solo pueden participar con voz en una de estas reuniones, realizada en agosto del 2005, invitadas para que puedan explicitar los fundamentos principales de su anteproyecto.

En esa oportunidad -sin desconocer los mecanismos legislativos dados en el tratamiento de esta ley-, las integrantes del MMC asistentes a la reunión de comisión plantean su desacuerdo en la forma de construcción de la política pública y el lugar dado a la sociedad civil, especialmente demandan ser partícipes protagónicas del proceso parlamentario, ya que son autoras del anteproyecto que puso en marcha la discusión sobre el tema en la legislatura.

A continuación, retomamos las propias palabras expresadas por las mujeres del Movimiento en la única reunión de comisión en la que pudieron participar,

"...También me parece pertinente aclarar que lo que estamos demandando, después de habernos tomado el trabajo de hacer una recopilación -que fue un trabajo muy arduo porque hubo que armar una base de datos con toda la legislación nacional e internacional-y de acercárselos a los legisladores, es que nunca se nos llamó para avisarnos que la ley iba a ser tratada y las veces que hemos venido a la Comisión lo hemos hecho a presión (...) Esto es lo que estamos demandando y lo hacemos en el sentido de que somos todos ciudadanos y como seguramente hay cosas para cambiar en esta sociedad una de ellas es la relación de los Poderes con la ciudadanía. Concretamente, ésta es la demanda que estamos haciendo: cómo mejorar este tipo de relación..."

(Patricia Trigueros, integrante del MMC)

"... Nosotras no tenemos muchas oportunidades para que se escuche nuestra voz, por lo que queremos aprovechar esta ocasión para decirles que es muy importante para ustedes enterarse de lo que le ocurre a la ciudadanía. A nosotras no sólo que no nos comunicaron que el bloque de la Unión Cívica Radical iba a tomar el proyecto sino que nos hubiera gustado que fuera tomado por todas las legisladoras que componen la Cámara. Por otro lado, recién el viernes, previo a un fin de semana largo, nos

10 Funcionamiento reconstruido a partir de las expresiones de las y los legisladores registradas en la versión taquigráfica de la reunión conjunta de las Comisiones de Solidaridad y Legislación General, Función Pública, Reforma Administrativa y Descentralización, Córdoba, 16 de agosto de 2005. Legislatura de la Provincia de Córdoba.

11 Realizamos esta observación, con relación a que hay versiones taquigráficas de ocho reuniones de Comisiones, y por tanto, constan la existencia de sus realizaciones. Sin embargo, según nos informaron empleados administrativos de la legislatura, no siempre hay registro taquigráficos de las reuniones, por lo que podrían haber existido mayor cantidad de reuniones. 
avisaron que debíamos asistir a esta reunión y nos enteramos esta mañana porque nos mandaron un mail..."

(Valentina Machietto, integrante del MMC)

"... En realidad, hace meses que estábamos tratando de tener voz porque también es necesario para nosotros poder aclarary decir qué pasa cuando uno trabaja tanto tiempo en conjunto, con muchísimas dificultades, y cómo se perciben las cuestiones de manera distinta cuando se está de un lado o del otro de las puertas de la Legislatura..."

(Alejandra Martín, integrante del MMC)

La escasa influencia en las políticas públicas por parte de movimientos y grupos de activistas se puede pensar con relación a la confluencia de múltiples intereses en el ejercicio del poder político, donde, como señala Duarte (2007), "se entremezclan las problemáticas del cuerpo social, objeto de la formulación de directrices de política, con las problemáticas del cuerpo o aparato político". La discusión sobre la problemática quedó, de alguna forma, "atada" al contexto del bipartidismo político presente en la legislatura de la provincia.

En esta misma reunión de Comisión, el MMC expone los puntos claves esgrimidos y argumentados del anteproyecto presentado: la necesidad de centrarse en la violencia contra las mujeres y las distintas formas de expresión de la misma; la consideración de las relaciones interpersonales además del ámbito familiar; y la importancia de las acciones de prevención y atención psicosociolegal. Dentro de este último punto, resaltan tanto la demanda de creación de un refugio permanente como la prevención de las revictimizaciones institucionales.

Luego de las exposiciones de las mujeres del movimiento, se realizan las intervenciones de los legisladores presentes ${ }^{12}$. En una de las intervenciones, el legislador Costa, del partido gobernante -Unión por Córdoba-, hizo referencia a un proyecto de violencia familiar presentado por los/as legisladores Arias, Vigo y Juncos, de su mismo partido político. Aboga por atender a este último proyecto señalando que fue presentado en primer término, y por tanto, va a la proa del debate, en un claro apoyo a su bancada. Desconoce así la presentación anterior del proyecto del MMC, sosteniendo además una argumentación que no hace referencia a los contenidos de los proyectos o su importancia social.

La otra intervención fue de la legisladora Gutiérrez, del Partido Nuevo, quien interrogó la necesidad de creación de un refugio permanente. Argumentó que la ley debería brindar las herramientas necesarias para que la exclusión del hogar sea del agresor y no del agredido, desconociendo lo ya expresado por el MMC en esa comisión, esto es, que se trataría de un refugio transitorio que atienda las situaciones de urgencias.

12 Si bien la propuesta de la Comisión fue que luego de la presentación de los puntos principales del anteproyecto, los/as legisladores/as realizaran preguntas o se tratara algún tema del que necesitan mayor información, llamativamente solo se registraron taquigráficamente dos intervenciones de los/as legisladores presentes. 
En ambos casos se revela una falta de escucha al planteo de las mujeres del MMC y un desconocimiento de las situaciones reales que cotidianamente viven miles de mujeres víctimas de violencia. La exposición del anteproyecto presentado por el MMC buscaba dar cuenta de los aspectos superadores y más abarcativos de los contenidos en el proyecto de Unión por Córdoba.

Finalmente, interesa recuperar algunas argumentaciones enunciadas por los/as legisladores/as en la sesión parlamentaria del $1^{\circ}$ de marzo de 2006, donde se aprueba la Ley No 9283.

En dichas argumentaciones se evidencian de manera explícita sentidos hegemónicos cristalizados acerca de la violencia familiar. Se insiste así en explicar la violencia familiar a partir de teorías psicológicas de sustrato mecanicista y con aparente sustento científico. Al respecto, los/as legisladores/as expresaron,

"... un niño que nacey se desarrolla en un hogar violento será un adulto maltratador..."

(Legisladora Alejandra Vigo, Unión por Córdoba)

"... no poner freno a la patología de la violencia familiar a tiempo significa otorgar como herencia a las generaciones venideras conductas violentas aprendidas..."

(Legisladora Isabel Biancciotti, Unión Cívica Radical)

".. .es conocido que las conductas violentas se transmiten por aprendizaje..."

(Legisladora Isabel Biancciotti, Unión Cívica Radical)

Mas allá de que dichas afirmaciones ya no se sostienen de manera lineal en la realidad cotidiana, lo preocupante es el sesgo psicologista que vuelve a situar el problema de la violencia exclusivamente dentro del ámbito intrafamiliar recayendo la responsabilidad en los sujetos que lo conforman e invisibilizando la construcción de la violencia como problemática histórica, social, cultural y política.

En la misma línea de sentido, se expresaron argumentaciones que ponen de relieve y refuerzan el lugar que debe ocupar la familia como garante social,

"... Este proyecto de ley es un primer paso hacia la solución de un tema más complejo, y parte de una premis a fundamental que nos permitirá trabajar formando convicciones y principios basados en la defensa de la vida y la familia..."

(Legisladora Ma. Ernestina Ramos, Partido Frente Nuevo)

"...para pelear por los derechos de la familia como célula insustituible de nuestra sociedad..."

"...que sean los hijos quienes alienten a sus padres para que concurran ante la justicia a hacer valer sus derechos para que nunca más una víctima de violencia familiar en Córdoba se sienta sola..."

(Legislador Alfonso Mosquera, Partido Córdoba Justicialista) 
"...por ello nos parece realmente halagüeño que hoy, en esta cámara, hayamos podido consensuar un proyecto que sirva para mejorar la calidad de vida de quienes son víctimas de violencia y para que definitivamente encontremos el camino de la tolerancia para la convivencia en el seno familiar..."

(Legisladora Ana Ma. Dressino, Unión Cívica Radical)

Nuevamente se focaliza en un aparente bienestar familiar donde se exalta la familia como célula básica de la sociedad, y se deniegan o invisibilizan los diversos ámbitos y relaciones interpersonales donde se produce y reproduce la violencia, así como la responsabilidad y lugar que le cabe al Estado ${ }^{13}$. De alguna manera, las enunciaciones realizadas en las sesiones parlamentarias responden a lo que Fraser (1991) denomina "discursos reprivatizadores", entendiendo por tales a aquellos discursos que buscan volver a contener las necesidades politizadas dentro de las interpretaciones tradicionales como es el discurso pro familia en este caso. Es necesario indicar que algunas y algunos representantes legislativos, en sus intervenciones, señalaron la necesidad de atender la violencia dirigida específicamente hacia las mujeres, aunque no llegaron a tener un tono cuestionador respecto de los discursos que ponían a la familia como preocupación principal.

En las sesiones parlamentarias que analizamos se puede observar la vigencia de la observación realizada por Dador Tozzini (2007), quien señala que los temas vinculados a la inequidad y discriminación de género no forman parte habitual de las posiciones o acuerdos partidarios adoptados previamente por las bancadas. En general, muchos de estos temas se consideran como temas de "conciencia", donde los y las representantes votan -y se expresan de forma anterior- sobre la base de sus convicciones personales, siendo posibles en mayor medida presiones por parte de los sectores más conservadores.

Luego de esta breve descripción del proceso y teniendo en cuenta la dinámica planteada entre el surgimiento de una iniciativa desde los movimientos de mujeres y feministas, el debate parlamentario y finalmente la sanción de una ley, nos importa analizar distintos sentidos respecto de la violencia hacia las mujeres que se ponen en discusión.

\section{PONIENDO EN TENSIÓN EL ANTEPROYECTO DEL MOVIMIENTO DE MUJERES CÓRDOBA Y LA LEY № 9283}

En este apartado presentaremos las diferencias que se presentan entre el "Anteproyecto de ley para prevenir, sancionar y erradicar la violencia hacia las mujeres en el ámbito familiar y las relaciones interpersonales", en tanto construido desde un movimiento social que pugna por incorporar los derechos plenos de las mujeres a una vida sin

13 Ana María Fernández (1993) refiere a la exaltación de algunos aspectos de la realidad y la denegación de otros, como un mecanismo que contribuye a la eficacia de los mitos sociales. 
violencia en una legislación, y la Ley Nº 9283 sobre Violencia Familiar -que efectivamente el Estado provincial sanciona.

En una lectura inicial, surge la primera distinción: los objetos-problemas a los cuales se refieren ambos documentos difieren sustancialmente. Mientras el MMC se propuso una ley que tomara por objeto la violencia hacia las mujeres, buscando concretar de ese modo la adhesión nacional a la Convención Interamericana para Prevenir, Sancionar y Erradicar la Violencia contra la Mujer -Convención de Belém do Pará-, la Ley No 9283 se suma a una serie de leyes provinciales que toman por objeto la violencia familiar en Argentina.

En la propuesta del MMC, la enunciación de Violencia hacia las mujeres visibiliza de forma clara a las mujeres como destinatarias de la violencia, una problemática denunciada como uno de los mayores flagelos de la humanidad, y que habitualmente está diluida o pensada en las políticas públicas dentro de la violencia familiar, es decir, circunscripta al ámbito de las relaciones familiares. El anteproyecto del MMC, además de tener en cuenta este ámbito, incorpora las relaciones interpersonales para legislar sobre la violencia hacia las mujeres. Explicita en detalle todas las acciones que se entienden por violencia hacia las mujeres, inclusive el mantenerla dentro de los roles sexuales estereotipados. Asimismo, la violencia aparece como una acción con una dirección, explicitando que son las mujeres y las niñas las personas objeto de la violencia, lo que tiene implicancias al momento de diseñar acciones de prevención, asistencia integral y erradicación de dicha violencia. El texto presenta así coherencia entre la definición que da sobre la violencia hacia las mujeres y el lugar de los sujetos involucrados en estas relaciones: quiénes ejercen violencia y quiénes la reciben.

En el caso de la Ley No 9283, la violencia se refiere al ámbito familiar, planteándola como un componente de dicho sistema y substrayéndola, de este modo, de un análisis que incorpore las relaciones de poder imperantes en nuestra sociedad entre los géneros y las generaciones. ${ }^{14}$ Asimismo, se omite la violencia económica en dicha definición, si bien se la incluye posteriormente en el artículo $5^{\circ}$ como una de las formas de violencia que se pueden dar, además de la física, psicológica o emocional. Por último, cuando se definen las situaciones y relaciones de los integrantes del grupo familiar en el Art. 40, se reduce el ejercicio de la violencia a las lesiones o malos tratos físicos o psíquicos, restringiendo así la propia definición de violencia dada en el Art. $1^{\circ}$.

No obstante estas marcadas diferencias, es significativo considerar el avance que produce la Ley de Violencia Familiar de la Provincia de Córdoba respecto de otras legislaciones provinciales, en lo que se refiere a la definición de grupo familiar,

14 Cap.1: Del Objeto, Art 30: "A los efectos de la aplicación de la presente ley, se entenderá por Violencia Familiar, toda acción, omisión o abuso dirigido a dominar, someter, controlar o agredir la integridad física, psíquica, moral, psicoemocional, sexual y/o la libertad de una persona en el ámbito del grupo familiar, aunque esa actitud no configure delito". 
entendiendo por tal “... el surgido del matrimonio, de uniones de hecho o relaciones afectivas, sean convivientes o no, persista o haya cesado el vínculo, comprendiendo ascendentes, descendientes y colaterales" (Art. 4º), haciéndose eco del anteproyecto del MMC que insistió permanentemente ante la legislatura provincial en la necesidad de ampliación de los sujetos activos que había que considerar en las situaciones de violencia.

Otro eje de análisis son los objetivos y acciones que se propone la ley, es decir, qué tipo de políticas públicas establece. En este punto, el Art. $33^{\circ}$ que crea el Programa de Erradicación de la Violencia Familiar, las indica como "políticas públicas de prevención y asistencia", aunque en la explicitación de las acciones desaparece la palabra "asistencia" y aparece el término tratamiento en distintos lugares del texto: "Determinar el daño sufrido por la víctima y aplicar el tratamiento adecuado para disminuir la trascendencia del mismo"; "Implementar el otorgamiento de un apoyo económico. . . bajo condición de que se sometan a tratamientos especiales..."; "Establecer tratamientos especiales de rehabilitación y reinserción, tanto para el agresor como para las víctimas" (los destacados son nuestros). Como se observa, hay una utilización recurrente del término tratamiento, y dicha palabra, debido a la representación que tiene, reduce el mismo al campo psicológico o al médico-psiquiátrico, con los efectos de individualización primero, y luego de posible psicologización de una problemática social. Se conforma así como una de las principales estrategias de asistencia, cerrando sentidos y posicionando además a la persona afectada en la misma situación de la persona agresora, e inclusive aparece el tratamiento como condición necesaria para otorgar un apoyo económico a la víctima. ${ }^{15}$

El carácter complejo de la violencia hacia las mujeres en el entorno familiar ha llevado a la formulación de diversos modelos teóricos, que buscan dar cuenta de su existencia y repetición a lo largo del tiempo y han dado sustento a abordajes específicos. Algunos modelos han centrado su explicación en variables individuales, psiquiátricas y biológicas; otros en la dimensión interaccional, relacional o vincular en el ámbito familiar; y por último, existen modelos que enfatizan el papel de la cultura y lo económico. Algunos autores han construido tipologías sobre los modelos teóricos: Gelles y Strauss (1978) han señalado teorías intraindividuales, teorías socioculturales, y teorías psicosociales; Lewis Okum (1987) identifica teorías de enfoque psicoanalítico, las basadas en la teoría del aprendizaje, otras derivadas de las teorías del control coercitivo, las feministas y las sociológicas. Emilio Viano (1992) reconoce las siguientes teorías: del intercambio, de la cultura de la violencia, del aprendizaje social, de los recursos, ecológica, evolucionista, sociobiológica, del conflicto social y de los sistemas generales. Jana Jazinki (2001), por

15 En el artículo 33 inciso f) se establece claramente una forma de coacción para el otorgamiento económico, en la medida que sostiene "Implementar el otorgamiento de un apoyo económico dinerario, no remunerativo ni reintegrable para que las personas afectadas puedan establecer su residencia temporaria en un lugar preservado del riesgo al que se encontraren expuestas, bajo condición de que se sometan a tratamientos especiales brindados por el equipo interdisciplinario que determine la reglamentación" (los destacados son nuestros). 
su parte, sistematiza las teorías de la siguiente forma: teorías microorientadas, teorías macroorientadas y teorías multidimensionales ${ }^{16}$.

Desde algunos de estos modelos se han construido interpretaciones e intervenciones "terapéuticas" que, en su mayoría, siguen desconociendo el carácter político de dicha problemática, que sí ha sido articulado por el movimiento feminista desde la década del 70. Bajo el lema lo personal es político, las feministas expresan y denuncian la dimensión social y política de la violencia hacia las mujeres.

Sin embargo, como mencionábamos, la modalidad de abordaje que se instrumenta desde la normativa provincial se enmarcaría dentro de teorías de la psicología individual, reconociéndose la incidencia de algunos factores sociales y culturales como desempleo, bajos niveles de educación, pobreza y adicciones. En efecto, desde el lenguaje construido por la normativa, la violencia sería una conducta patológica, desviada y disfuncional, de una persona psíquicamente perturbada -el victimario-; y la víctima tendría algún tipo de daño psíquico y/o rasgo patológico por el que se mantiene en un vínculo enfermo.

El tratamiento dispuesto para víctimas y victimarios se reduciría a una terapia psicológica a nivel individual y grupal.

Respecto de la víctima, habría un reconocimiento y “corrección" de estos rasgos patológicos que darían sostén a los hechos violentos y a nivel grupal se buscaría reforzar las capacidades para enfrentar las situaciones de violencia y no "permitir" que se repitan.

Con el victimario, la terapia individual tendría los objetivos de controlar los impulsos biológicos y situaciones de estrés que sufren los hombres; como se expresa en un folleto de difusión del Grupo Terapéutico de Hombres de la Dirección de Violencia Familiar: "si te pasó esto... sudor, nerviosismo, temblores, verborragia, no poder pensar, no registrar lo que hacés... existe un lugar donde te pueden ayudar, controlar la ira es posible, pide ayuda". Se desliza de esta afirmación que la conducta violenta del victimario es expresión de una situación de estrés.

Las mujeres son reducidas a la condición de víctimas; focalizando en la imagen de mujeres dañadas y reforzando así los estereotipos culturales. En la práctica se instituye un experto con conocimiento fáctico sobre cómo la mujer "debe ser"y "qué debe hacer" para evitar hechos violentos en su hogar; al instalar modelos de comportamiento funcionales y generales, negando las capacidades de agencia y la diversidad de las mujeres. "Cuando la víctima es concebida como débil y necesitada, la categoría en símisma crea dificultades porque somete a la víctima a una relación binaria donde ella es endeble, y tanto el victimario como quien debe ayudarla es el fuerte" (Truño Salvado, 2007: 141). Y como dice Villavicencio, se desconoce que "todas las víctimas están constantemente

16 Estas distintas clasificaciones se encuentran sistematizadas en MDGF (2010). 
tomando medidas para evitar que el agresor las maltrate" (2001: 36, citado por Marugán Pintos y Vega Solís, 2002: 419).

En otro sentido, Alfonso Rodríguez González, desde un enfoque de derechos humanos, y rescatando los aportes realizados en un Foro Virtual sobre el concepto de víctima a nivel internacional ${ }^{17}$, señala que la categoría víctima aparece

"como una forma de resistencia activa, de ejercicio de derechos civiles con el fin de evitar la impunidad y la desmemoria, reconociendo y reconociéndose no solo en el sufrimiento, sino también y especialmente en la condición de actores sociales -por tanto, sujetos de derechos-, en procura de que se haga justicia, se reparen los daños ocasionados y se garantice la no repetición de las violaciones". (Rodríguez, 2008, p. 37).

En este contexto, la noción de víctima se dispone estratégicamente para denunciar las agresiones, visibilizar la desigualdad, y como táctica para promover la equidad entre hombres y mujeres.

Es interesante remarcar que en el anteproyecto del MMC se utiliza el término Atención psico-socio-legal, ubicando en el mismo plano de importancia los tres tipos de atenciones e intervenciones, aunque no se explicita qué se entiende por Atención.

En cuanto a las políticas de prevención y asistencia en violencia, la Convención de Belem do Pará obliga a los Estados firmantes a reconocer los derechos de las mujeres en todos los ámbitos de sus relaciones y garantizar la accesibilidad a los servicios de atención y justicia como parte de esos derechos, y da lugar específicamente a medidas de prevención, señalando los ámbitos socioculturales, la educación y los medios de comunicación como espacios a trabajar e intervenir. Con relación a este punto, la Ley No 9283 propone como Prevención: "la promoción de una cultura que favorezca la creación de un marco objetivo de equidad, libertad e igualdad entre los miembros de una familia, eliminando las causas y patrones conductuales que generan y refuerzan la violencia familiar", la que resulta de una generalidad tal que impide pensar cuáles son las acciones necesarias para transformar la cultura actual ni quiénes son los principales responsables de esa prevención. Luego, en la especificación del Programa de Erradicación de la Violencia Familiar (Art. 33 Inc. i), se plantea que se busca promover la función de prevención y asistencia en organizaciones de la sociedad civil, apareciendo

17 En este Foro participaron profesionales de España y Latinoamérica, en donde el debate se centró en la reflexión sobre los términos de sobreviviente, damnificado y víctima, para poder dar cuenta de líneas de abordaje coherentes con una perspectiva en derechos humanos. Varias intervenciones señalaron las connotaciones de debilidad, desventaja y minusvalía que la noción de víctima tiene a nivel social simbólico, pero reconocen que a nivel jurídico representa una política de reparación por parte del Estado. Sin embargo, advierten la necesidad de visibilizar los encargos por parte del Estado, ya que en el uso de la noción de víctima también se explicitan las necesidades de "reparar" el sufrimiento individual, desvinculando la problemática de su dimensión política. Plantearon el desafío de contar con modelos de abordaje que se centren en la capacidad de transformarse de los sujetos, dispositivos que dispongan al encuentro con el otro y evitar los procesos de re-victimización a nivel institucional: "que los sujetos puedan trascender y ser capaces de construir proyectos de vida y convertirse en actores sociales de procesos tan importantes para la colectividad como son la búsqueda de verdad, justicia y reparación" explicitó una de sus participantes, Carolina Baltodano (Grupo de Acción Comunitaria, 2008). 
así cierta delegación de la responsabilidad del Estado respecto de este tipo de acciones. A posteriori, en el Decreto que crea el Programa, el papel de las organizaciones no gubernamentales son reconocidas solamente en la tarea de sensibilización para la toma de conciencia de las víctimas. Esto resulta, de alguna forma, una subvaloración del papel de la sociedad civil y específicamente de las organizaciones feministas y de mujeres en la política pública. Dichas organizaciones han estado comprometidas históricamente con el derecho de las mujeres a una vida sin violencia, ya que además de realizar acciones de acompañamiento y asesoría a mujeres en situaciones de violencia, han desarrollado debates e intervenciones públicas respecto de este tema, y han exigido a los organismos estatales responsables rendición de cuentas sobre las políticas desarrolladas. El Estado, por medio del Programa creado, se hará cargo entonces solamente de la asistencia.

Contrariamente, en el anteproyecto presentado por el MMC, la prevención es pensada como una de las funciones y responsabilidades principales que debe realizar el "Programa de prevención, atención y erradicación de la violencia hacia las mujeres" que se propone, involucrando responsabilidades específicas de los Ministerios de Educación, Salud, Secretaría de Justicia y Derechos Humanos. La prevención no se escinde de la asistencia y la erradicación de la violencia en las acciones propuestas, si bien no se avanza en el documento respecto de mayores explicitaciones de qué se entiende por tal.

Por último, interesa señalar que el anteproyecto especifica al menos tres acciones particulares de gran interés que no se encuentran presentes en el texto de la Ley Provincial No 9283: 1) La coordinación de distintos programas y servicios de prevención y asistencia; 2) Medidas respecto de la responsabilidad de los medios de comunicación; 3) Creación de un refugio permanente de asistencia transitoria.

\section{EL PROCESO DE INSTITUCIONALIZACIÓN DE LA LEY PROVINCIAL № 9283. UN ANÁLISIS DE LA NORMATIVA SANCIONADA}

En marzo del 2006 se sanciona, como dijimos anteriormente, la Ley No 9283 -Ley de Violencia Familiar. La Reglamentación de la Ley Nº283 se expidió en el año 2007 -a un año de sancionada dicha ley- donde se incluye la reglamentación del Programa de Erradicación de la Violencia Familiar de la provincia de Córdoba. Y recién en junio del 2008, mediante el Decreto 1030/08, se crea dicho Programa al mismo tiempo que su Consejo Consultivo, el Observatorio y el Registro provincial, así como la Ampliación del "Programa Nueva Vida" (Decreto 1340/03) que brinda una asignación económica. Iremos analizando cada uno de estos documentos como parte del proceso de institucionalización de la Ley No 9283.

La reglamentación de Ley determina -en su Anexo A- como objeto de la misma la prevención, detección temprana y atención de la Violencia Familiar; y define las competencias y jurisdicciones, denuncia y procedimiento judicial para el abordaje de la problemática. Se establece un marco preventivo, que incluye la prevención, detección 
temprana y atención de la violencia, subrayando así la prevención primaria que abarca prácticas integrales en los ámbitos institucional, organizacional y comunitario, la prevención secundaria que atiende al círculo familiar y a las personas involucradas en hechos de violencia, y la prevención terciaria que refiere a la instrumentación de la asistencia a las víctimas o aquellas personas con posibles factores de riesgo.

Entendemos que este abordaje da cuenta de un paradigma de salud pública basado en un modelo epidemiológico clásico, con el foco puesto en los factores de riesgo individuales. En este sentido, las víctimas deberán ser detectadas, diagnosticadas y tratadas, desestimando en el abordaje los aspectos políticos, sociales, culturales y económicos que configuran la complejidad de la problemática de violencia.

Ahora bien, si analizamos la Reglamentación del Programa de Violencia Familiar, tanto las acciones específicas que se nombran como los recursos humanos destinados se orientan a la atención y tratamiento de la víctima, con carácter fundamentalmente psicológico, en detrimento de los demás objetivos enunciados de prevención, capacitación e investigación.

En efecto, el acento está puesto en el ámbito de la asistencia, como atención específica tendiente a apoyar a las personas frente a la violencia familiar, en la esfera psicológica, asesoría legal y asistencia social. Para el abordaje de la problemática se determina, en un primer nivel, la atención telefónica, y en un segundo nivel, la atención directa interdisciplinaria de los sujetos involucrados en violencia familiar, en la cual intervienen cuatro áreas. El Área Administrativa recepta la demanda, recibe y diligencia los oficios judiciales, maneja el archivero y paga las "becas" de asistencia económica. El Área Social realiza el primer contacto con la persona o institución que demanda, materializando dicha entrevista en un formulario. En casos particulares se recurre a la interconsulta. Por último, el Área Psicológica brinda psicoterapia individual y grupal para las personas que sufren y los que ejercen violencia. La terapia individual se recomienda en casos de situaciones de alto riesgo, estado de confusión severo, angustia y fobia, violación, hijas/os abusadas/os. El Área Legal asesora sobre dispositivos y acciones judiciales a realizar, realiza atención jurídica requerida por turnos o guardia, y acompañamiento a Tribunales.

Por otro lado, y respecto del ámbito de la Prevención solo se define la conformación de una Red, en la que participarían los Ministerios de Salud, de Educación, de la Solidaridad, de Seguridad y las áreas de Mujer y Niñez, e instituciones y organizaciones públicas y privadas de la capital y del resto de la provincia.

Atendiendo al Decreto 1030/08 que crea el Programa de Prevención y Erradicación de la Violencia Familiar en la provincia de Córdoba, encontramos también explicitada la situación de desventaja en la que se halla la víctima "objeto de este flagelo", que es la violencia familiar. En efecto, la violencia así entendida conlleva a que las personas que "la padecen" deben ser "tratadas", deslizándose el sentido de enfermedad y reforzando la idea de pasividad. 
En este sentido, se resaltan medidas que se instrumentan con el fin de procurar la integridad física, psicológica, y económica de la víctima y la inserción laboral de víctimas y victimarios, así como profundizar el seguimiento de los casos en los que ha habido deserción en el tratamiento. Asimismo, se considera como catalizadores de violencia el alcoholismo y la drogadicción, para lo que se dispondrá de las medidas necesarias y adecuadas para quienes padecen dichas adicciones.

Para permitir la correcta "individualización de las personas afectadas"y para el resguardo de la confidencialidad de los datos, se plantea crear un Registro Provincial de violencia familiar, a fin de procesar, analizar y producir estadísticas para la formulación de políticas.

Este Decreto protocoliza los deberes, facultades, autorizaciones yámbitos de aplicación de los diferentes organismos e instituciones que participan en la implementación del referido programa ${ }^{18}$. Crea además el Observatorio para la erradicación de la Violencia Familiar y el Consejo Consultivo de Violencia Familiar, en el cual participarían los tres poderes estatales provinciales, iglesias y confesiones reconocidas, universidades públicas y privadas, organizaciones no gubernamentales con trayectoria en la problemática, así como colegios y consejos profesionales.

Es significativo señalar que el Decreto, si bien enuncia en las consideraciones iniciales que la violencia es una violación a los derechos humanos fundamentales, caracterizándola explícitamente "como un problema político, social, cultural y de salud pública que demanda respuestas y recursos públicos para su prevención y asistencia", vuelve a recortar la complejidad de la problemática de la violencia a la relación de dos personas: la agredida y la agresora, diluyéndose el lenguaje de los derechos.

El objetivo que señala el Programa es la atención integral, para la cual se conforman equipos interdisciplinarios. En contradicción con esto, lo que efectivamente se reglamenta es el tratamiento desde la terapia psicológica de forma individual y grupal, a los efectos de "fortalecer" capacidades individuales para la toma de decisiones y la disminución de riesgos, básicamente señalada como la no convivencia con el agresor en el caso de las mujeres. Situaciones que reducen el sentido de violencia de género a una problemática individual, como aparece en la definición de asistencia dada en la Reglamentación del Programa: "se entiende como tal a la atención específica, tendiente a apoyar a las personas en un proceso de cambio". Fraser ya había señalado, en el caso norteamericano, que las demandas de los movimientos feministas respecto de la necesidad de generar recursos económicos y sociales para la independencia de las mujeres -como genérico social- se sustituyeron en la administración estatal por "un enfoque más estrecho, sobre los problemas femeninos de la "baja autoestima'" (1991: 29).

18 El Ministerio de Justicia mediante la Dirección de Violencia Familiar debe reforzar la interrelación y articulación con los Ministerios de Desarrollo Social, Educación, Salud, Obras Públicas, Secretarías de Niñez; de Cultura, General de la Gobernación y las Agencias de Deportes, cámaras empresariales, entidades sindicales y organizaciones no gubernamentales, para la erradicación de la violencia familiar. 
Por otra parte, en el proceso de institucionalización se va acentuando el carácter excluyente de víctimas que deben ser atendidas que otorga la normativa a las mujeres, sin la necesaria apelación en simultaneidad de su carácter de ciudadanas con derechos, tal como lo hemos analizado anteriormente. Ejemplo de ello son las condiciones de admisión al Programa de las personas víctimas de violencia familiar ${ }^{19}$. Las víctimas de violencia pueden ingresar al Programa, luego de ser certificados como tal para la obtención de los "beneficios", que refieren fundamentalmente a la atención psicológica gratuita y a una asignación económica mensual ${ }^{20}$. En caso de incumplimiento se dispondrá la suspensión de los "beneficios" que hubieran sido otorgados, previa intimación. Cabe llamar la atención sobre la denominación que tiene dicha asignación económica, la cual es calificada como "Beca de asistencia en situación de crisis".

Hablar de beneficios y por tanto, de beneficiarios, en estas circunstancias, es al menos, paradójico, y al mismo tiempo, posibilita una operación de desalojo de ciudadanía y del lenguaje de los derechos. Como nos recuerda Fraser (1991), las prácticas enunciatorias no son insignificantes a la hora de evaluar la construcción de particulares "modos de subjetivación" en los miembros de los grupos subordinados en su interacción con las políticas estatales.

Virginia Guzmán (2001) señala que ha sido más fácil legitimar los problemas de género desde el discurso de la vulnerabilidad y no desde el discurso sustentado en el reconocimiento y ejercicio de los derechos de las mujeres. La autora señala que "el rechazo a la violencia contra las mujeres es coherente con aquellos esquemas interpretativos que las consideran sujetos de protección" (2001: 27). Podríamos suponer que el énfasis en "contar las muertas" realizado por los movimientos feministas ha logrado ser un discurso más aceptado por la sociedad civil y el Estado, en tanto ancla en representaciones y creencias acerca de las mujeres como sujetos que deben ser tutelados y protegidos. Esto, señalan Guzmán (2001) y Vargas (2009), ha posibilitado que en la región fueran más fácilmente promulgadas leyes respecto de la violencia hacia las mujeres, siendo más difícil la aprobación de aquellas que afirman el protagonismo político y social de las mujeres, tales como las leyes referidas a derechos sexuales y reproductivos.

\section{CONCLUSIONES}

El Movimiento de Mujeres Córdoba, como expresión organizativa del movimiento feminista y de mujeres de la provincia de Córdoba, buscó generar una ley que

19 "Tendrán derecho a ingresar al programa, las personas víctimas de violencia familiar que acrediten tal calidad a través de certificado expedido por la autoridad de aplicación de este Programa, en el que se deje constancia que dicha persona debe recibir alguno de los beneficios indicados en el presente, y que se encuentre en situación socioambiental que así lo justifique. Las personas que ingresen al programa deberán sostener el proceso de tratamiento terapéutico indicado" (Anexo Único del Decreto 1030/08).

20 El Programa Nueva Vida estipulaba una suma no integrable, ni remuneratoria por 24 (veinticuatro) meses de $\$ 500$ (quinientos pesos) en el año 2010. Inicialmente, cuando se creó dicho Programa en el año 2003, se entregaba una suma de $\$ 200$ por solamente seis meses. 
comprometiera al Estado provincial en su conjunto en la atención de una problemática de derechos humanos: la violencia hacia las mujeres señalando el contexto sociocultural de desigualdad entre los géneros como productor y sostenedor de dicha violencia.

Así, y como producto de esta interacción, podemos observar que la normativa provincial que se construyó y sancionó respecto de la violencia familiar, vino a cubrir algunos vacíos legales y de procedimiento en referencia a la problemática de la violencia hacia las mujeres en el ámbito familiar. Al incorporar parcialmente algunos aportes del anteproyecto de Ley del MMC, se ubicó en un lugar de avanzada respecto de otras leyes provinciales de violencia familiar del país, al incluir las diversas formas de violencia y contemplar una definición amplia de grupo familiar, así como la del sujeto "activo" de la violencia al incluir no convivientes.

Sin embargo, realizó un desvío central a los ojos del movimiento de mujeres: la ley y la política se abocaría - una vez más- a la violencia familiar, subsumiendo y restringiendo la violencia hacia las mujeres al interior de aquella. Fraser señala en este punto, que

"los movimientos sociales buscan medidas estatales para resolver sus necesidades 'fugitivas' al mismo tiempo que se oponen a las interpretaciones terapéuticas y administrativas. Así, estos ejes significan conflictos entre interpretaciones rivales de las necesidades sociales y construcciones rivales de identidad social" (Fraser, 1991, p. 26).

La normativa cordobesa centra el trabajo de la unidad de aplicación de la misma -el Programa de Violencia Familiar - en la asistencia, y dentro de la misma, a la asistencia psicológica, definida como "tratamiento", dándose entonces lugar a un enfoque terapéutico, y por ende, la violencia aparecería como enfermedad del grupo familiar, de los vínculos que establecen las personas. Asimismo, la relación a las distintas formas de prevención se inscribe dentro de un paradigma sanitarista, y no de derechos humanos, definiendo de algún modo la preeminencia de algunas profesiones y de algunos enfoques en los equipos interdisciplinarios.

Este tipo de abordaje descontextualizaría el problema de la violencia, al considerarlo de orden individual-privado, despolitizando el sufrimiento. Es decir, por un lado, se desconoce la emergencia de la problemática en contextos sociales específicos; se naturaliza la violencia y se deshistorizan los procesos sociales que ponen en evidencia que la subordinación de la mujer es una realidad que se traduce en diferentes experiencias de abuso físico, psicológico, económico en la cotidianeidad de estas; donde el principio de igualdad y el derecho a la vida no se cumple ni simbólica ni materialmente.

Al decir de Mireya Suárez,

"... los actos violentos, como también los derechos específicos que violan, no están contenidos naturalmente en ningún lenguaje ni en ninguna sociedad, por lo que la tarea de identificarla y construir el acto violento es tan incesante como lo es la marcha de la región y los cambios que experimenta constantemente" (Suárez, 2004, p. 13). 
Finalmente, es necesario advertir que en nuestro análisis hemos trabajado casi exclusivamente con los documentos de la normativa sancionada. Si bien la normativa guía las prácticas institucionales llevadas a cabo, no conforma en sí misma la política pública desarrollada sobre la violencia hacia las mujeres en la provincia de Córdoba. Las prácticas institucionales concretas pueden consolidar o desestabilizar los sentidos presentes en la normativa. El análisis de dichas prácticas forma parte de la próxima etapa de nuestra investigación.

Sin embargo, el análisis realizado de las definiciones y procedimientos que se consignan en las normativas nos permiten sostener que la política provincial respecto de la violencia hacia las mujeres no escapa a las deficiencias que presentan políticas similares, que se resumen en lo que plantean Marugán Pintos y Vega Solís (2002) cuando sostienen que el abordaje de la violencia por el Estado español, mediante el "gobierno de la emergencia y de la excepcionalidad", implica desconocer la violencia hacia las mujeres como problema político, depositando las iniciativas de resolución en las víctimas y las/os especialistas.

El enfoque de derechos humanos propone en cambio, como explicita Guendel González, que

\begin{abstract}
"las políticas públicas para la atención de estas problemáticas deben incidir directa y explícitamente - no solo en la atención a la víctima- sino en el tejido social y familiar, lo cual requiere el desarrollo de iniciativas que propicien el papel activo de la sociedad, principalmente en el nivel local, desarrollando acciones de vigilancia, prevención o reincorporación de las víctimas (restitución de derechos). Esto implica algo más que el 'apoyo funcional' de la comunidad y de la familia, obligando a articularlas en procesos mismos de formulación y gestión de la política" (Guendel González, 2002, p. 124).
\end{abstract}

Los esfuerzos de los movimientos feministas en lograr convenciones internacionales que obliguen a los Estados a garantizar los derechos de las mujeres a una vida sin discriminación ni violencia, como han sido la CEDAW y la Belém do Pará, siguen sin tener suficiente eco en las políticas estatales.

Entendemos que las políticas públicas respecto de los derechos de las mujeres deben otorgar un lugar protagónico a los movimientos feministas y de mujeres, a sus argumentaciones y reivindicaciones, tanto en su discusión y diseño como en su implementación, evaluación y monitoreo, protagonismo que dista mucho de ser eficaz todavía en nuestras realidades nacionales y locales.

Como diría Giulia Tamayo, "urge un feminismo que repolitice el malestar de las mujeres" (2010: 33), como la única forma de resistir políticas que insisten en modos de gestión de la violencia de género atrapados en un lenguaje burocrático y terapéutico, donde las mujeres -abordadas como casos individuales- son consideradas pasivas beneficiarias de políticas y no sujetos titulares de derechos. 


\section{BIBLIOGRAFÍA}

Abramovich, Víctor (2006):"Una aproximación al enfoque de derechos en las estrategias y políticas de desarrollo". Revista de la CEPAL No88. pp. 35-50. [on line] Disponible en: http://www.eclac.cl/publicaciones/xm//2/24342/G2289eAbramovich.pdf

Bosch Fiol, Esperanza y Ferrer Pérez, Victoria (2000):"La violencia de género: de cuestión privada a problema social". Intervención Psicosocial: Revista sobre lgualdad y Calidad de Vida. Vol. 9 No 1. pp. 7-19.

Comité D'Action Femmes et Sécurité Urbaine-CAFSU (2002): La Seguridad de las mujeres: De la Dependencia a la Autonomía. Actuando para la seguridad de las mujeres. Montreal, Canadá.

Chejter, Silvia (2009): Conferencia presentada en la Mesa Redonda "Políticas Públicas orientadas a la prevención y el tratamiento de las violencia de género". $1^{\text {er }}$ Congreso Interdisciplinaria sobre Género y Sociedad. PIEMG-CIFFYH y PG-SEU. Universidad Nacional de Córdoba. Córdoba, 27 de mayo.

Dador Tozzini, María Jeannie (2007): “Contexto político de la región: desafíos y oportunidades para las políticas de género", en Falú, Ana y Segovia, Olga (Edit.), Ciudades para convivir: Sin violencia hacia las mujeres, pp. 41-60. Santiago de Chile: Edic. Sur.

Duarte, Eliana (2007): Usos políticos de la diferenciación étnica y racial. Un estudio de la circunscripción electoral especial. Institute for Research and Debate on Governance [on line]. Disponible en: http://www.institut-gouvernance.org/en/analyse/ficheanalyse-292.html

MDGF - Fondo de las Naciones Unidas y España para el cumplimiento de los objetivos de desarrollo del milenio (2010): Estudio sobre tolerancia social e institucional a la violencia basada en género en Colombia. Bogotá.

Ezpeleta, Cecilia (2007): "La fuerza de la sociedad civil y el diálogo social". Sociodisea. Revista de enfoque crítico del derecho y la justicia penal. Año V. No 8. Nov. Dic. 2007, pp. 24-27.

Fernández, A. M. (1993):"De lo imaginario social a lo imaginario grupal", en Fernández, A.M y De Brassi, J. (Comp.), Tiempo histórico y campo grupal, pp. 69 - 91. Buenos Aires: Ed. Nueva Visión.

Fraser, Nancy (1991):"La lucha por las necesidades. Esbozo de una teoría crítica socialista feminista de la cultura política del capitalismo tardío". Debate Feminista, México, pp. 3-40.

Grupo de Acción Comunitaria (2008): Foro on line en el I Congreso Internacional Construyendo Redes. Abril 2008. 
Guendel González, Ludwig (2002): "Políticas públicas y derechos humanos". Revista de Ciencias Sociales, Año/Volumen III, No 097. Universidad de Costa Rica, pp. 105-125. [on line] Disponible en: redalyc.uaemex.mx/pdf/153/15309709.pdf

Guzmán, Virginia (2001): "La institucionalidad de género en el Estado: nuevas perspectivas de análisis". Serie Mujer y Desarrollo No 32. Santiago de Chile: Unidad Mujer y Desarrollo. CEPAL. Naciones Unidas. [on line] Disponible en: http://www.eclac.org/ publicaciones/xml/3/6323/lcl1511e.pdf

Markez, Alonso; Fernández Liria, A.; Pérez-Sales, P. (eds.) (2009): Violencia y salud mental. Salud mentaly violencias institucional, estructural, socialy colectiva. Valladolid: Asociación Española de Neuropsiquiatría. Estudios.

Marugán Pintos, Begoña y Vega Solís, Cristina (2001): "El cuerpo contra-puesto. Discursos feministas de la violencia". [on line] Disponible en: http://www.sindominio.net/karakolal antigua_casa/cuerpocontrapuesto.htm.

Marugán Pintos, Begoña y Vega Solís, Cristina (2002): "Gobernar la violencia. Apuntes para un análisis de la rearticulación del patriarcado". Política y Sociedad, vol. 39, 2, pp. 415-435.

Pateman, Carole (2005): El contrato sexual. Barcelona: Edit. Anthropos - Universidad Autónoma Metropolitana-Iztapalapa.

Provoste, Patricia (2007): "Violencia contra la mujer en la pareja: respuestas de la salud pública en Santiago de Chile". Serie Mujery Desarrollo No 85. Santiago de Chile: CEPAL. Naciones Unidas.

Rainero, L.; Rodigou, M.; Pérez, S. (2005): Herramientas para la promoción de ciudades seguras desde la perspectiva de género. Córdoba: Edic. CISCSA.

Rodigou, Maite (2011): "Violencia hacia las mujeres: entre la visibilización y la invisibilización", en A.A.V.V. Derechos Humanos, Género y Violencia, pp. 77-78. Córdoba: SEU-UNC y Ministerio de Justicia de la Provincia de Córdoba.

Rodríguez González, Alfonso (2008): El concepto de víctima. Mesa Redonda El Concepto de Víctima. I Congreso Internacional Construyendo Redes. Grupo de Acción Comunitaria. [on line] Disponible en: http://documentacion.aen.es/pdf/libros-aen/ coleccion-estudios/violencia-y-salud-mental/parte1-algunos-conceptos-sobre-laviolencia/37-el-concepto-de-victima.pdf.

Sagot, Montserrat (2008): "Estrategias para enfrentar la violencia contra las mujeres: reflexiones feministas desde América Latina". Athenea Digital, 14, p. 215-228. Disponible en http://psicologiasocial.uab.es/athenea/index.php/atheneaDigital/article/view/571 
Segato, Rita (2003): Las estructuras elementales de la violencia. Ensayos sobre género entre la antropología, el psicoanálisis y los derechos humanos. Bs. As: Edit. Universidad Nacional de Quilmes.

Suárez, Mireya (2004): Provocando la reflexión sobre el discurso "violencia contra la mujer". Texto para Discusión. Brasilia, UNIFEM / LAC.

Tamayo León, Giulia (2010): “Violencia de género, seguridad ciudadana y políticas públicas: revisión de los deberes estatales de prevención y garantías de los derechos humanos de las mujeres y las niñas". Memorias del Seminario Internacional "Mujeres Seguras en las Ciudades Futura. Hacia una vida sin violencia de género: visiones y propuestas", pp. 27-44. D.F., México: Comisión Nacional para Prevenir y Erradicar la Violencia contra las Mujeres.

Vargas, Virginia (2009): "La violencia de género: pistas para un análisis", en Falú, Ana (Editora) Mujeres en la ciudad. De violencias y derechos, pp. 55-60. Santiago de Chile: Red Mujer y Hábitat de América Latina y Edic. Sur. 